\title{
Mode-locked fiber laser with a fiber phase modulator
}

\author{
M. W. Phillips and A. I. Ferguson \\ Department of Physics, The University, Southampton S09 5NH, UK
}

\author{
G. S. Kino and D. B. Patterson
}

Department of Electrical Engineering, Ginzton Laboratories, Stanford University, Stanford, California 94305

Received January 30, 1989; accepted April 25, 1989

FM mode-locked operation of a single-mode $\mathrm{Nd}^{3+}$-doped fiber laser has been achieved with an integrated fiber phase modulator. The technique permits a low-loss cavity configuration, resulting in low threshold and high slope efficiency. Pulses of $\sim 200$-psec duration are observed at a repetition rate of $417 \mathrm{MHz}$ with an average output power of $15 \mathrm{~mW}$.

We present a novel approach to active mode locking of a single-transverse-mode fiber laser with an integrated fiber phase modulator. This low-threshold system can be diode pumped and has a bandwidth capable of supporting subpicosecond-duration pulses. Potential miniaturization along with low fabrication costs lends the system to a variety of applications, including laser range finding, electro-optic sampling, and time-resolved spectroscopy.

For a given dopant ion the transition linewidth is much broader in a glass host than in a crystalline host. Glass-fiber lasers are therefore suitable candidates for tunable- and short-pulse operation. Rare-earthdoped fiber lasers have the added feature of being lowthreshold systems owing to guidance of pump and laser beams in the fiber gain medium. We recently demonstrated FM mode locking of a $\mathrm{Nd}^{3+}$-doped fiber laser, using a bulk electro-optic phase modulator $\left(\mathrm{LiNbO}_{3}\right)$ placed intracavity close to the output coupling mirror. ${ }^{1}$ This laser emitted bandwidth-limited sech $^{2}$-type pulses of 20-psec duration and 1-W peak power.

A major limitation of this arrangement was the requirement for intracavity optical elements. This both increased intracavity loss and severely restricted the lasing bandwidth through étalon formation. Geister and Ulrich ${ }^{2}$ have reported FM mode locking of a fiber laser; they used an integrated-optic $\mathrm{LiNbO}_{3}$ :Ti phase modulator. This was directly butted to one end of the doped fiber, preventing étalon formation, but losses were considerable because of a mismatch between the fiber and modulator transmission modes. Here we describe FM mode locking of a similar laser system, but this time we adopt intrafiber phase modulation, that is, direct modulation of the phase of the fiber mode's electric field. This technique simplifies laser cavity design, removing the limitations referred to above.

A schematic diagram of the mode-locked laser is shown in Fig. 1. The active medium of the laser was a 24.55-cm length of $\mathrm{Nd}^{3+}$-doped, non-polarizationmaintaining fiber with the following characteristics: numerical aperture N.A. $=0.21$, second mode cutoff wavelength $\lambda_{\text {c.o. }}=945 \mathrm{~nm}$, and a dopant concentration of 220 parts in $10^{6} \mathrm{wt}$. Nd. It was therefore single mode at the lasing wavelength of $1.09 \mu \mathrm{m}$. The peak response frequency of the modulator (maximum phase retardation) was $\nu_{\max }=417.0 \mathrm{MHz}$. The optical path length of fiber required to match this to the optimum mode-locking frequency was $35.72 \mathrm{~cm}$, corresponding to a free-running intermode beat frequency of 419.9 $\mathrm{MHz}$. This mismatch in passive cavity mode spacing and repetition rate for optimum mode locking has been attributed to dispersion effects of the lasing $\mathrm{Nd}^{3+}$ ions. $^{2}$ The fiber ends were cleaved to a flatness tolerance of less than $1.6 \mu \mathrm{m}$ over the fiber diameter. One end was hard-butted to a thin substrate mirror (width, $0.17 \mathrm{~mm}$ ). This permitted $>85 \%$ transmission at the pump wavelengths used, $820 \mathrm{~nm}$ (AlGaAs laser diode) and $590 \mathrm{~nm}$ (R6G dye laser), while being highly reflecting $(R=99.5 \%)$ at the laser wavelength, permitting efficient end-pumping of the gain medium. The output end was similarly hard-butted to an output coupling mirror with a laser transmission of $15 \%$. This mirror was wedged and antireflection coated on the rear surface to suppress multiple reflections.

The pump beam was launched into the fiber through the high reflector, using a standard $10 \times \mathrm{mi}$ croscope objective (N.A. 0.25). At the output end, laser emission was collected and collimated after the output coupler with an $18 \times$ microscope objective (N.A. $=0.35)$ with a transmission of $92 \%$.

The phase modulator was an acousto-optic device pressed firmly against the outside surface of the fiber.

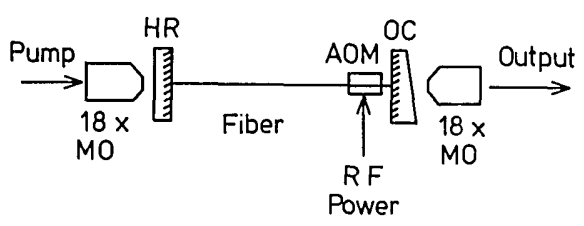

Fig. 1. Schematic diagram of FM mode-locked fiber laser: $\mathrm{HR}$, high reflector; OC, output coupler; MO, microscope objective; AOM, acousto-optic modulator. 


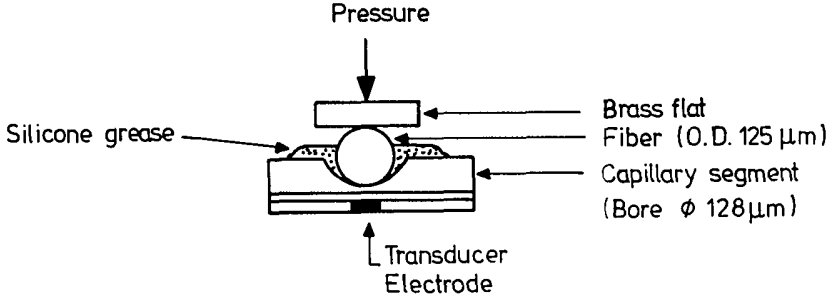

Fig. 2. Fiber/modulator arrangement transverse to the fiber axis.

It was located close to the output coupling mirror to maximize the mode-coupling efficiency $\delta .^{3}$ A general description of the fabrication and operational characteristics of the device is given elsewhere. ${ }^{4}$ Figure 2 shows a schematic of the modulator arrangement transverse to the fiber axis. The fiber is an integral part of the modulator, which is a standing-wave device with resonances determined by the fiber cladding diameter. The uncoated fiber section rested in the bore of a half-capillary, polished to 1-mm thickness with a $\mathrm{ZnO}$ transducer layer, with electrodes arranged on the undersurface as described in Ref. 4. Acoustic waves set up in the capillary were coupled into the fiber through an impedance-matching agent (silicone grease) while pressure was exerted on the fiber from above with a clamped brass flat. The bore diameter of $128 \mu \mathrm{m}$ required a cladding diameter of $125 \pm 1 \mu \mathrm{m}$ to ensure a good Hertzian contact for efficient coupling of acoustic energy into the fiber. The active length of the modulator was $4 \mathrm{~mm}$.

At the resonance center frequency of $417 \mathrm{MHz}$, the acoustic wavelength in the fiber was $\sim 13 \mu \mathrm{m}$. This was sufficiently larger than the $2-\mu \mathrm{m}$ mode spot size in the fiber, so that sinusoidal phase modulation occurred for standing-wave patterns symmetric about the core region. The maximum single-pass phase retardation, $\delta_{m}$, was measured in situ by monitoring sideband generation on a single-frequency He-Ne laser beam $(\lambda=633 \mathrm{~nm})$ launched into the fiber. Figure 3 shows the variation in $\delta_{m}$ with applied rf power, $P_{\mathrm{rf}}$. We see that for $\delta_{m}$ measured in radians and $P_{\mathrm{rf}}$ measured in watts, the following relationship holds:

$$
\delta_{m} \simeq 1.1 \sqrt{P_{\mathrm{rf}}}
$$

The resonance bandwidth (FWHM) was $\sim 2 \mathrm{MHz}$, centered near $417 \mathrm{MHz}$. Consequently, the physical fiber length of $24.55 \mathrm{~cm}$ had to be cleaved to a tolerance of $0.5 \mathrm{~mm}$ to ensure good matching of the optimum mode-locking frequency $\nu_{\mathrm{ML}}$, with the peak phase-retardation frequency $\nu_{\max }$. In this particular case, $\nu_{\mathrm{ML}}=416.9499 \mathrm{MHz}( \pm 200 \mathrm{~Hz})$ coincided exactly with $\nu_{\text {max }}$. Allowing for the inverse dependence of $\delta_{m}$ with wavelength, we assume a 0.6 -rad phase shift for $1 \mathrm{~W}$ of rf power applied at the optimum modelocking frequency.

The low-loss nature of the system was reflected in its excellent $\mathrm{cw}$ lasing characteristics. With dye-laser pumping, the threshold pump power incident upon the launch objective was $2.5 \mathrm{~mW}$. With an overall launch efficiency $\eta_{L}=0.37$ and absorption efficiency $\eta_{\mathrm{ABS}}=0.95$, this corresponds to just $900 \mu \mathrm{W}$ of ab- sorbed pump power. The output power of the laser was $15 \mathrm{~mW}$ for $35 \mathrm{~mW}$ of absorbed pump power, indicating a slope efficiency, with respect to absorbed power, of $\eta_{\mathrm{SL}}=0.44$. With diode pumping, the threshold pump power incident at the launch objective was $4 \mathrm{~mW}$. The laser output power was $700 \mu \mathrm{W}$ for an incident pump power of $15 \mathrm{~mW}$. With a fiber dopant concentration selected for dye-laser pumping, absorption at $820 \mathrm{~nm}$ was poor, with an efficiency $\eta_{\mathrm{ABS}}$ $=0.41$. The launch efficiency was $\eta_{L}=0.32$. Thus, with respect to absorbed pump power, the threshold power was just $520 \mu \mathrm{W}$ with a slope efficiency of 0.49 . Mode locking the laser with $1 \mathrm{~W}$ of applied rf power had a negligible effect on the threshold and slope efficiency.

The following results were obtained with dye-laser pumping at $590 \mathrm{~nm}$ with an absorbed pump power of $35 \mathrm{~mW}$ (although similar results for pulse width and lasing profile were obtained with diode pumping). With correctly butted mirrors, modulating the phase at frequency $\nu_{\text {ML }}$ yielded two sets of pulses, each corresponding to either extremum of the phase modulation. With pulse durations of $\sim 400$ psec and a separation of $1.2 \mathrm{nsec}$ the output signal was nearly sinusoidal. By withdrawing the output coupler by a few micrometers, an étalon was introduced that controlled the lasing spectral profile while maintaining low cavity loss. This permitted selection of a single set of mode-locked

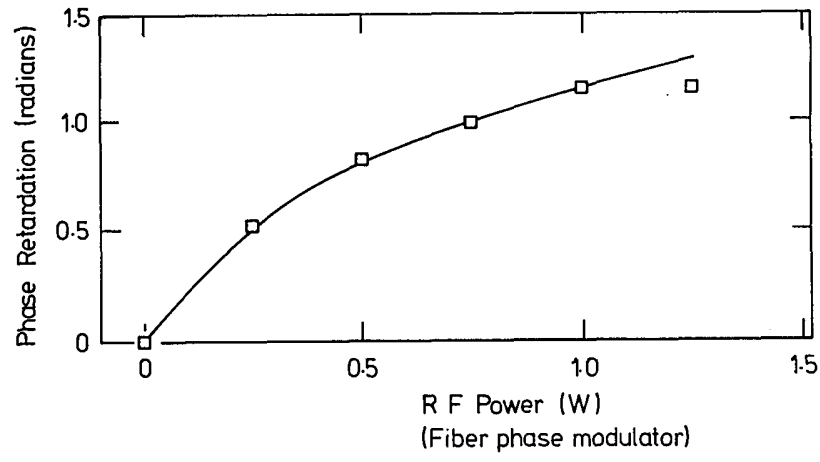

Fig. 3. Phase retardation versus rf power (measured at 633 nm). Fitted curve: $\delta_{m}=1.1 \sqrt{P_{\mathrm{rf}}}$.

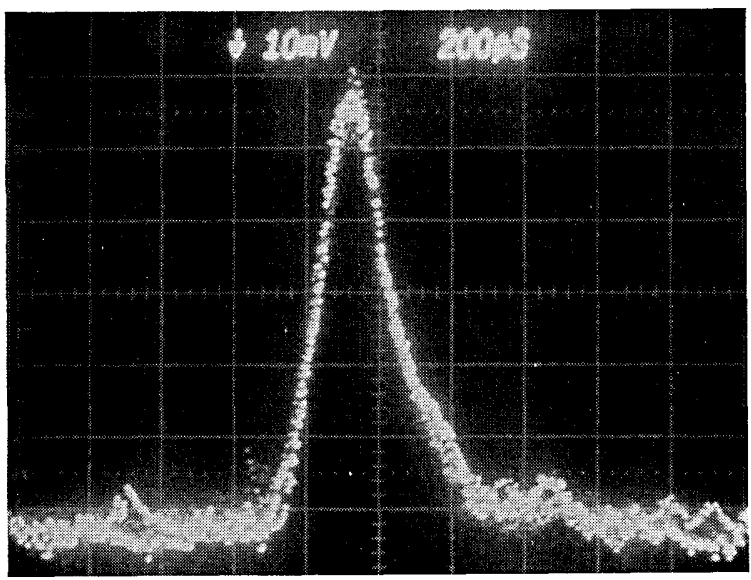

Fig. 4. Sampling oscilloscope display of FM mode-locked pulses from a fiber laser, detected by a InGaAs photodiode (combined detector response time, $50 \mathrm{psec}$ ). 


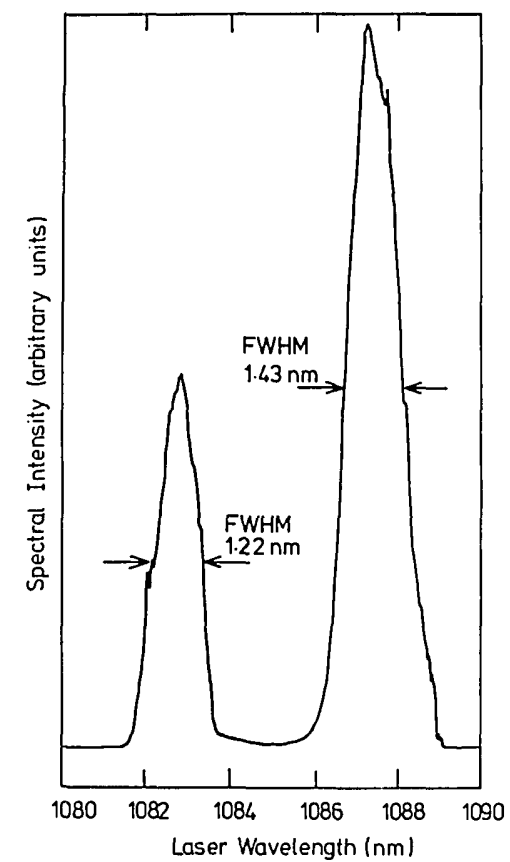

Fig. 5. Power spectrum of FM mode-locked laser (monochromator resolution, $0.2 \mathrm{~nm}$ ).

pulses with a 200-psec pulse duration and a repetition rate of $\sim 417 \mathrm{MHz}$. Figure 4 is an oscilloscope trace of the pulse profile monitored with a fast InGaAs photodiode and a sampling scope (combined detection response time, 50 psec). The long trailing edge of the pulse suggests insufficient mode coupling over the lasing bandwidth. A significant change in pulse width was observed for $200-\mathrm{Hz}$ steps in modulation frequency.

A plot of the power spectrum of the mode-locked laser is given in Fig. 5. The measurement was made with a $0.33-\mathrm{m}$ monochromator with $0.2-\mathrm{nm}$ resolution. It shows two peaks separated by $5 \mathrm{~nm}$, each with a bandwidth of $\sim 1.5 \mathrm{~nm}$ (FWHM). Pulses are clearly not bandwidth limited, and a means of bandwidth restriction is necessary to suppress the trailing edge of the pulse.

We have demonstrated a simple technique for producing FM mode-locked pulses in a single-transversemode fiber laser. The cavity design has low-loss characteristics and permits broadband laser operation. Pulses have a 200-psec duration (FWHM) at a repetition rate of $417 \mathrm{MHz}$ with a peak power of $180 \mathrm{~mW}$. The power spectrum is double peaked with $1.5-\mathrm{nm}$ bandwidths. We expect that shorter pulses can be achieved by restricting the laser bandwidth to some optimum value for the phase retardation available. This is under investigation and will be reported in due course.

This research has been partially funded within the Joint Opto-Electronics Research Scheme. We are grateful to the Optical Fibre Group of the Department of Electronics, Information Engineering and Computer Science, The University, Southampton, for the provision of fiber. The research of M. W. Phillips is supported by a Science and Engineering Research Council studentship.

\section{References}

1. M. W. Phillips, A. I. Ferguson, and D. C. Hanna, Opt. Lett. 14, 219 (1989).

2. G. Geister and R. Ulrich, Opt. Commun. 68, 187 (1988).

3. S. E. Harris and O. P. McDuff, IEEE J. Quantum Electron. QE-1, 245 (1965).

4. D. B. Patterson, A. A. Godil, G. S. Kino, and B. T. KhuriYakub, Opt. Lett. 14, 248 (1989). 